Rev Soc Esp Dolor

2014; 21(3): 185

\section{Hacia la perspectiva GRADE (II)}

\section{Sr. Director:}

Es necesario dar más detalles de la clasificación GRADE, que traducida al español significa: "Clasificación de la Evaluación, Desarrollo y Valoración de las Recomendaciones", la cual fue expuesta en esta sección de la revista, publicada en ediciones anteriores (1) del presente año.

El grupo de trabajo GRADE nace en el año 2000 con el objetivo de abordar las deficiencias de los actuales sistemas de clasificación de la evidencia. Este grupo ha elaborado una clasificación que presenta un enfoque sistemático y explícito a partir de la definición de una serie de criterios necesarios, para hacer juicios sobre la calidad de la evidencia y fuerza de recomendación. Estos criterios consisten en los siguientes puntos:

1. Definir target group: consiste en definir nuestro público objetivo, o problema de interés, el tipo de intervención que nos interesa y su comparación (no es obligación que exista una comparación).

2. Resultados de interés (outcome): debemos seleccionar los desenlaces de interés y valorar su implicancia clínica. Para llevar a cabo este ítem es necesario realizar un listado de 9 resultados de interés clínico dentro de los cuales se subdividen en: "no importante 1-3", "importantes 4-6" y "críticos 7-9" (es fundamental mencionar que los resultados "no importantes 1-3" no se utilizan para entregar las recomendaciones).

3. Valorar la evidencia: esto consiste en que debemos tener claridad en la magnitud del efecto y en su calidad para cada uno de los resultados seleccionados como "críticos e importantes" -se debe considerar que los estudios clínicos aleatorizados (ECA) presentan el nivel más elevado de la jerarquía de la evidencia en terapia, seguido de los estudios observacionales-. Este ítem finaliza con la evaluación global de la evidencia donde encontramos 4 puntos (2):

- Alta calidad: es poco probable que investigaciones adicionales cambien nuestra confianza en la estimación del efecto. Hay suficientes datos con intervalos de confianza estrechos.

- Moderada calidad: es probable que investigaciones adicionales tengan un impacto importante en la confianza de la estimación del efecto y puedan cambiar la estimación.

- Baja calidad: es muy probable que investigaciones adicionales tengan un impacto importante en la confianza de la estimación del efecto y es probable que cambie.

- Calidad muy baja: presenta una gran incertidumbre sobre la estimación del efecto.

- Ninguna evidencia: no hay evidencia de ECA.

4. Beneficios/riesgo/costes: una vez valorada la evidencia, tenemos que centrarnos en los posibles beneficios, riesgos y costes de la intervención; esto quiere decir que debemos decidir si el balance entre los beneficios/riesgos y costes son positivos y viables para poder justificar la decisión (no olvidar que debemos respetar siempre los valores y preferencias de los pacientes).

5. Dirección y fuerza de la recomendación: en la metodología GRADE se diferencian dos categorías relativas hacia la dirección de la recomendación: (a favor o en contra de la utilización de la intervención) y también relativa hacia la fuerza de recomendación (fuerte y débil), presentando cuatro categorías: fuerte a favor; débil a favor; débil en contra; y fuerte en contra.

6. Se finaliza con la redacción de las recomendaciones y su justificación.

R. Aguilera Eguía ${ }^{1,2}$, E. Zafra Santos ${ }^{3}$, C. Rojas Sepúlveda ${ }^{4}$, G. Aguayo Alcayala ${ }^{4}$ y D. Sánchez León ${ }^{4}$

\author{
${ }^{1}$ Musculoskeletal Group Cochrane Colaboration; \\ Member of the Grade Working Group; Member of the \\ Cochrane Trainer's Network, Colaboración Cochrane \\ Iberoamericana, Red Chilena de Salud Basada en \\ Evidencia. ${ }^{2}$ Universidad Santiago de Chile. Chile. \\ ${ }^{3}$ Escuela de Kinesiología. Universidad Santo Tomás. \\ Santiago de Chile, Chile. ${ }^{4}$ Licenciados en Kinesiología. \\ Santiago de Chile, Chile
}

\section{BIBLIOGRAFÍA}

1. Aguilera R, Zafra E, Pavez G, Rojas C, Aguayo G, Sánchez D, Aguilera T. Niveles de evidencia y grados de recomendación (I). Hacia la perspectiva GRADE. Rev Soc Esp Dolor 2014;21(2):92-6.

2. Higgins JPT, Green S, editors. Cochrane Handbook for Systematic Reviews of Interventions. Version 5.0.1 [Updated September 2008]. The Cochrane Collaboration 2008. Available at: www.cochrane-handbook.org 